University of Wollongong

Research Online

Faculty of Informatics - Papers (Archive)

Faculty of Engineering and Information

Sciences

21-11-2004

\title{
Role of wavelet transforms in image restoration
}

Prashan Premaratne

University of Wollongong, prashan@uow.edu.au

I. Burnett

University of Wollongong, ianb@uow.edu.au

Follow this and additional works at: https://ro.uow.edu.au/infopapers

Part of the Physical Sciences and Mathematics Commons

\section{Recommended Citation}

Premaratne, Prashan and Burnett, I.: Role of wavelet transforms in image restoration 2004.

https://ro.uow.edu.au/infopapers/168

Research Online is the open access institutional repository for the University of Wollongong. For further information contact the UOW Library: research-pubs@uow.edu.au 


\title{
Role of wavelet transforms in image restoration
}

\author{
Abstract \\ Image processing techniques including image restoration rely heavily on discrete Fourier transform (DFT) \\ for frequency domain representation for analysis of its frequency content. Even though wavelet analysis \\ has been around for more than a decade, much of its potential as a tool to analyze time-frequency \\ localization of signal has not been properly tapped. In blind iterative deconvolution where a degraded \\ image is restored with minimum a priori information about the original image or the point spread function \\ (PSF), it is almost impossible to evaluate whether a restoration is achieved or not without human \\ observation. We propose a new approach using wavelet decomposition to assess an image being \\ restored or not automatically.

\section{Keywords} \\ deconvolution, discrete Fourier transforms, image representation, image restoration, iterative methods, \\ time-frequency analysis, wavelet transforms

\section{Disciplines} \\ Physical Sciences and Mathematics

\section{Publication Details} \\ This paper originally appeared as: Premaratne, $\mathrm{P}$ and Burnett, I, Role of wavelet transforms in image \\ restoration, TENCON 2004. IEEE Region 10 Conference, 21-24 November 2004, vol A, 243-246. Copyright \\ IEEE 2004.
}




\title{
ROLE OF WAVELET TRANSFORMS IN IMAGE RESTORATION
}

\author{
Prashan Premaratne and Ian Burnett \\ School of Electrical, Computer \& Telecommunications Engineering, The University of \\ Wollongong, North Wollongong, NSW Australia 2500.
}

\begin{abstract}
Image processing techniques including image restoration rely heavily on Discrete Fourier Transform (DFT) for frequency domain representation for analysis of its frequency content. Even though Wavelet analysis has been around for more than a decade, much of its potential as tool to analyze time-frequency localization of signal has not been properly tapped. In blind iterative deconvolution where a degraded image is restored with minimum apriori information about the original image or the point spread function (PSF), it is almost impossible to evaluate whether a restoration is achieved or not without human observation. We propose a new approach using wavelet decomposition to assess an image being restored or not automatically.
\end{abstract}

\section{INTRODUCTION}

Ever since the Fast Fourier Transform (FFT) algorithm developed hand in hand with the emergence of the faster computers, FFT has been the tool of choice for frequency domain analysis for multidimensional signals. Although DFT is capable of decomposition of a signal to its spectrum, it lacks the ability to localize this information in time. In other words, DFT can not break the signal down to reveal what frequencies exist when (for one dimensional signals) and where (for two dimensional signals, i.e., images). However, with wavelet decomposition, this DFT short coming is alleviated and has been widely used in frequency analysis for more than a decade. Many have reported successful wavelet based coding techniques $[1,2,3]$ and wavelet based denoising techniques for both audio and video signals. There has been many wavelet based video coders that outperforms mpeg2 standard for video coding. The denoising techniques offer so much versatility to this new tool [4]. However, we have not seen any wavelet applications in image restoration in literature. One reason being that Discrete Wavelet Transform (DWT) transforms a two dimensional signal into four dimensional signal and unless the wavelet bases are properly chosen to be orthogonal, there would be redundant data that increases the computational complexity which is undesirable [1]. In blind image restoration $[5,6,7]$ and especially in iterative blind image restoration [5, 7], a human observer is a must to evaluate the progress of the restoration. That is to say, an observer has to evaluate the quality of the image being processed in order to decide whether further improvements are possible or the restoration is to be abandoned. In reality, the available information may never be sufficient to achieve any convergence and the process has to be abandoned after a great effort. In our previous attempts, we have come up with some performance quality measures that attempted to automate the process $[7,8]$. In this article, we demonstrate a novel way to use wavelet decomposition to sub sample and image so that in this abstract form, some useful information can be extracted to make automatic decision making without user/observer intervention. In the next section on theoretical developments we present wavelet decomposition and its purported use in iterative restoration analysis. This is followed by experimental results and finally the conclusions.

\section{THEORETICAL DEVELOPMENTS}

The wavelet transform is similar to a Fourier transform in the sense that it decomposes a signal into a set of basis functions. In the case of the Fourier transform this basis consists of sine and cosine functions at different frequencies. In the wavelet transform the basis function is

$$
\psi_{a, b}(x)=\frac{1}{\sqrt{a}} \psi\left(\frac{x-b}{a}\right), \quad a \in \mathbf{R}^{+}, b \in \mathbf{R}
$$

where each wavelet function in the decomposition is formed from a mother wavelet function $\psi(x)$ which is scaled (given by $a$ ) and translated $(b)$, the result being localised in both the frequency and spatial domains [9]. For two dimensional signals, the conventional DWT may be regarded as equivalent to filtering the input signal with a bank of band pass filters, whose impulse responses are all approximately given by scaled versions of a mother wavelet. The scaling factor between adjacent filters is usually two to one (2:1), leading to octave bandwidths and centre frequencies that are one octave apart. At the coarsest scale, a low pass filter is also required to represent the lowest frequencies of the signal. In other words wavelet analysis is a version of widowing technique, but with varying window size. It allows the use of longer windows when more precise low 
frequency information is required and shorter windows where high frequency information is needed. WT in wavelet analysis is analogous to the Fourier Transform in Fourier analysis.

We used Daubeshie's type 1 wavelet basis [9] for our analysis due to their simplicity. As it would become clearer, the choice of the wavelet basis functions has no impact on our restoration.

\section{Image Degradation Model}

Let the true (original) image be denoted by $f(x, y)$, the common PSF be given by $h(x, y)$ and the blurred image is denoted by $g(x, y)$. The blurring process can be expressed as

$$
\begin{gathered}
g(x, y)=f(x, y)^{*} h(x, y)+n(x, y), \\
0 \leq x, y \leq N+M-2
\end{gathered}
$$

where $n(x, y)$ denotes additive Gaussian noise in the system, * denotes 2-D convolution operator and the true images are of size $N \times N$ and PSF is of size $M \times M$. Since we assume the mean value of the true image is preserved in the degradation process the following expression holds,

$$
\left|\sum_{(l, m) \in R_{h}} h(l, m)\right|=1,
$$

where $R_{\mathrm{h}}$ is the support size of the PSF.

An image of 'Lenna' (Figure 1) of size $512 \times 512$ is degraded using a $5 \times 5$ PSF whose power spectrum is shown in Figure 2.

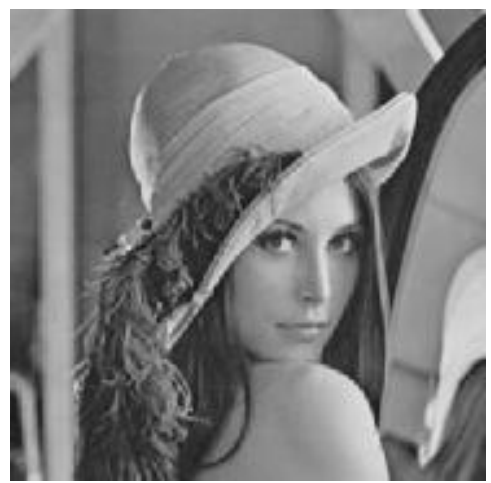

Figure 1. Original Image of Lenna

We perform level 1 decomposition the original image using Daubeshie's type 1 wavelet as shown in Figure 4. From this, it is obvious that detail levels include mainly higher frequencies that give rise to edges. Figure 5 shows similar decomposition done on degraded image in Figure 3. This information will be used in our automatic evaluation to be discussed next section.

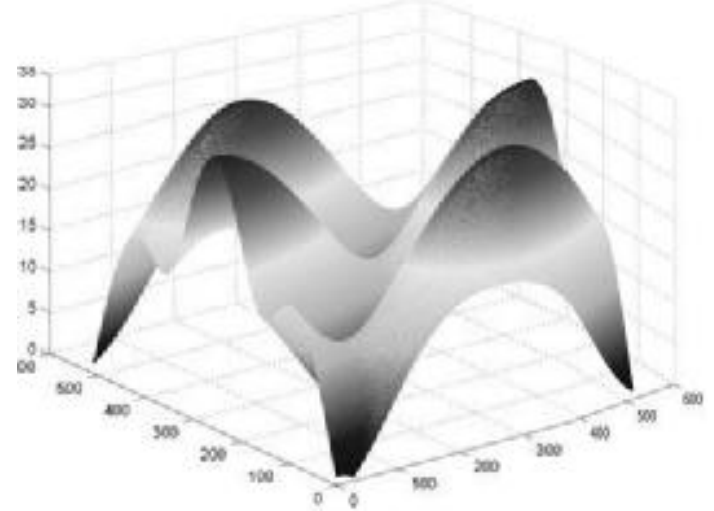

Figure 2. Power Spectrum of 5x5 PSF

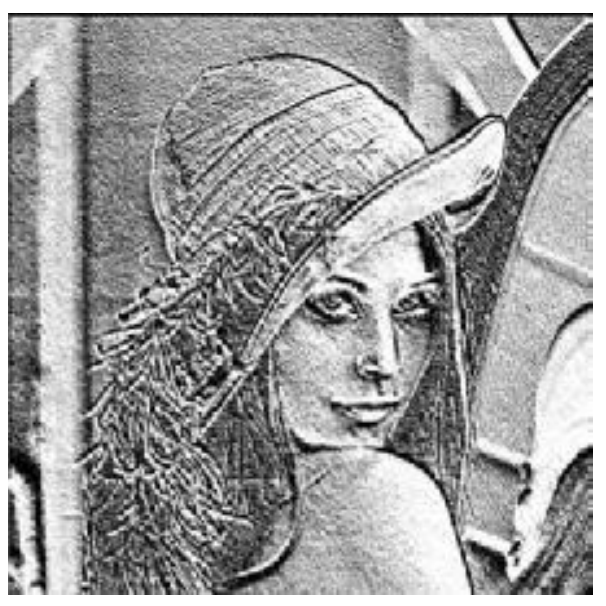

Figure 3. Degraded Image of Lenna

If we allow the upper case letters represent the 2-D Discrete Fourier Transform (DFT) of their lower case counterparts and BSNR is high such that we can ignore noise terms.

$$
G(k, l)=F(k, l) H(k, l), \quad 0 \leq k, l \leq N+M-2 .
$$

In this algorithm, initial estimate of the PSF is obtained using the concepts of zero sheet separation [7]. Assuming that an initial estimate of the PSF is available and the blurred images are corrupted with moderate amount of noise, we outline the next steps involved in the algorithm as follows.

The basic structure of the algorithm is presented in Fig. 6 . The image estimate is denoted by $\hat{f}(x, y)$ and the capital letters represent fast-Fourier transformed (FFT) versions of the corresponding signals. Subscripts denote the iteration number of the algorithm. 
Using the initial estimate of FFT of PSF, $\hat{H}(u, v)$, can be obtained using a guess or using zero sheet separation techniques [7], an initial estimate of the true image is calculated using the following recursive formula (Fourier constraint I in Fig. 6) [7]

$$
\tilde{F}_{1}(u, v) \approx\left[\frac{1}{\tilde{H}(u, v)} \frac{|\tilde{H}(u, v)|^{2}}{|\tilde{H}(u, v)|^{2}+K}\right] G(u, v),
$$

where $K$ denotes a constant which approximates the noise-to-signal power ratio. This can be arbitrarily chosen as this information may not be available in blind deconvolution scenario. After the initial estimate of the original image, successive estimates are calculated using the following incremental Wiener-type filter (Fourier constraint II in Fig. 6) [7].

$$
\tilde{F}_{k}(u, v)=\frac{G(u, v) \tilde{H}_{k-1}^{*}(u, v)}{\left|\tilde{H}_{k-1}(u, v)\right|^{2}+\alpha /\left|\tilde{F}_{k-1}(u, v)\right|^{2}}
$$

where $(.)^{*}$ denotes the complex conjugate of (.). The real constant $\alpha$ represents the energy of the additive noise and is determined by prior knowledge of the noise contamination level, if available. Since the algorithm is targeted at restoration of gray scale images, the intensity levels are distributed in a range from 0-1. Any negative values or positive values above this range are supposed to saturate the image sensor and hence could be used as a spatial domain constraint for the estimated image. Hence, the initial estimate of the image calculated from Eq. (4) is used in Eq. (5) for evaluating successive iterations of the true image using the following constraint

$$
\hat{f}(x, y)=\left\{\begin{array}{cc}
1, & \hat{f}(x, y)>1 \\
0, & \hat{f}(x, y)<0 \\
\hat{f}(x, y), 0 \leq \hat{f}(x, y) \leq 1
\end{array}\right.
$$

is used.

\section{Automatic Restoration Evaluation}

Now instead of user intervention to determine the progress of the restoration, the following scheme can be used. As indicated in Fig. 5, image details of degraded image are fuzzy in nature where as original image detail contain contours of the original image edges. This difference can be exploited for automatic image evaluation such that edge map of degraded image is compared with horizontal detail. Referring to Fig. 6, one can select different initial PSF estimates using different schemes and the iterative restoration can be run without user intervention so that a close match between the edge map and detail level map is achieved, indicating acceptable restoration.

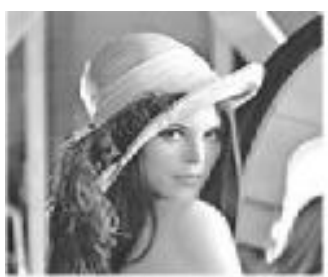

Approximation A1

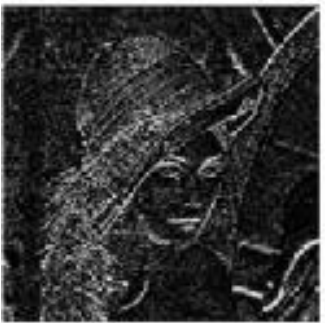

Vertical Detail o1

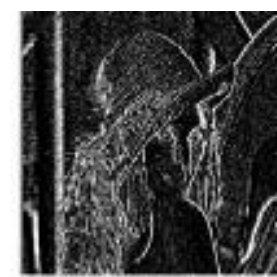

Honzontal Dotai HI

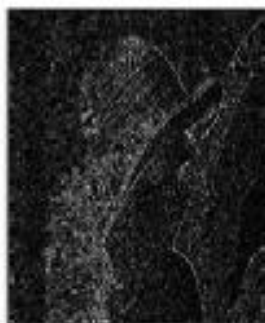

Diagonal Detail 01
Figure 4. Level 1 Wavelet Decomposition of Lenna
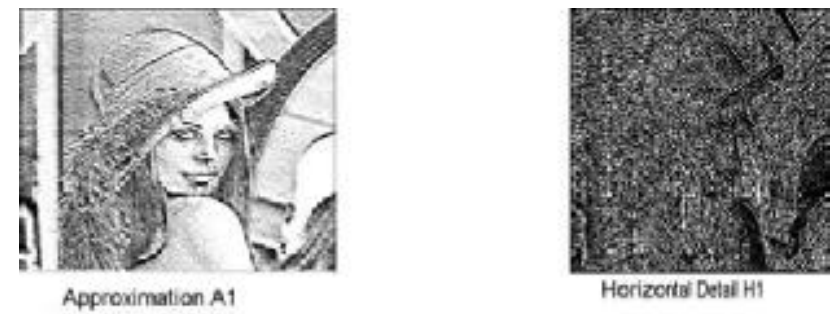

Horizonta' Delal HI
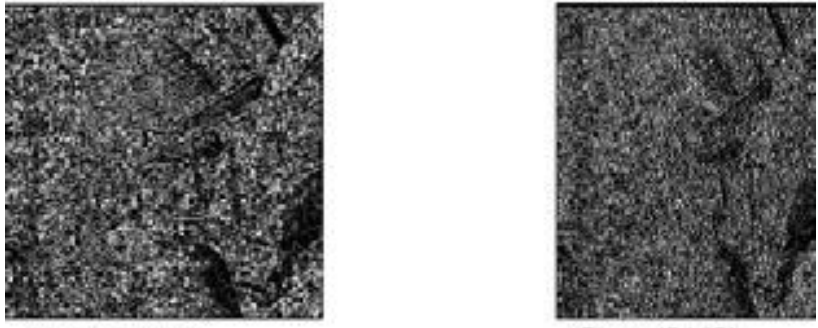

Vertical Detail V1

Figure 5. Level 1 Wavelet Decomposition of Degraded Lenna Shown in Figure 3. 


\section{EXPERIMENTAL RESULTS}

We used the degraded image shown in Figure 7, of well known 'cameraman' image to run the iterative restoration process. We used two initial estimates of PSF, one estimated using zero sheet techniques and the other a random PSF of size $5 \times 5$. Due to the nature of the Blind Image Deconvolution, we can not assume any other knowledge except the support size of the PSF (in this case). Using this iterative technique, we restored images using 12 and 376 iterations for zerosheet technique and random PSF selection in the absence of noise. The recovered images are shown in Figure 8.

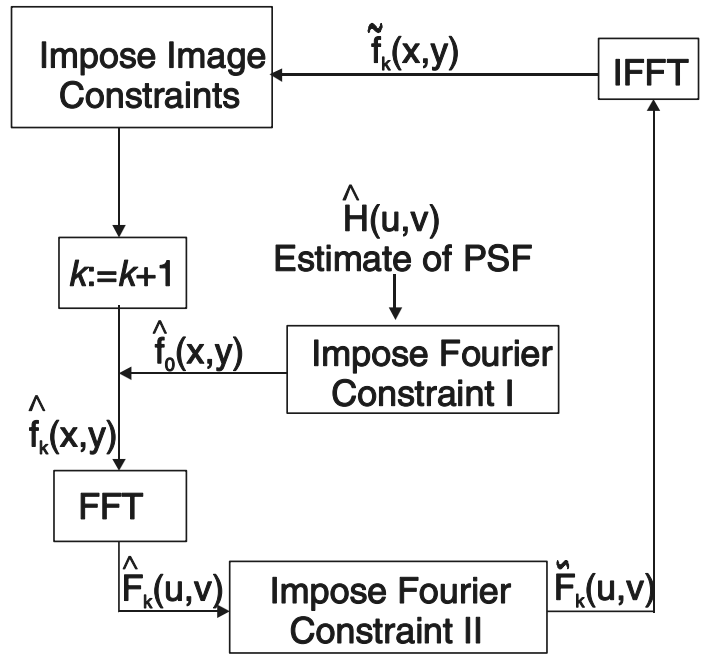

Fig. 6 Diagram of the Restoration Scheme

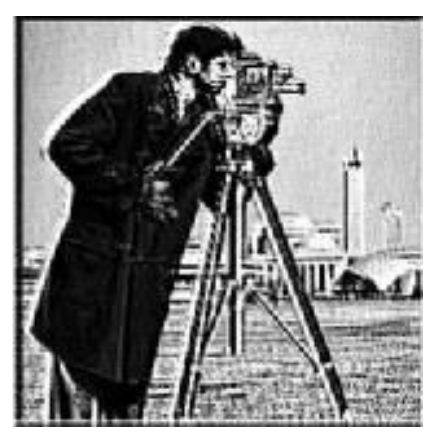

Figure 7. Degraded ‘Cameraman’ Image

\section{CONCLUSIONS}

From our experimental results, we can conclude that DWT can be used in other image processing applications apart from compression and denoising. We successfully applied it to automatic image evaluation using correlation to stop (when convergence achieved) iterative image restoration process which usually demands very high user intervention. This approach should be further extended to other image processing tasks to tap the enormous potential of DWT.

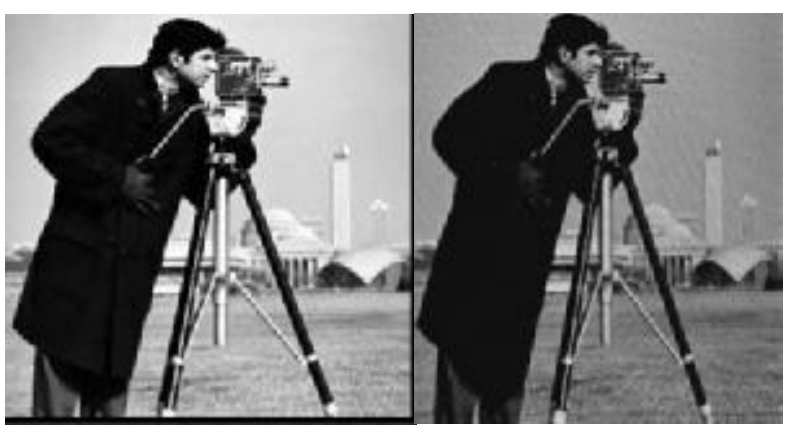

Figure 8. Recovered Images; Using Zerosheet Initial PSF (left), Using a 'guess' for Initial PSF (right)

\section{REFERENCES}

[1] .M. Shapiro,"Embedded Image Coding Using Zerotrees Of Wavelet Coefficients", IEEE Transactions on Signal Processing, vol. 41, No. 12 , pp. 3445-3462, 1993.

[2] C.D. Creusere, “ A New Method Of Robust Image Compression Based On The Embedded Zerotree Wavelet Algorithm" IEEE Transactions on Image Processing, vol. 6, No. 10 , pp. 1436-1442, 1997.

[3] V.R. Algazi, and R.R. Estes, Analysis Based Coding Of Image Transform And Subband Coefficients" Proceedings of the SPIE, vol. 2564, pp. 11-21, 1995.

[4] D.L. Donoho, "De-Noising by Soft Thresholding", IEEE Tans. Info. Theory, vol. 41-3, pp. 613-627, 1995.

[5] G. R. Ayers, J. C. Dainty, "Iterative Blind Deconvolution Method and Its Applications", Optics Letters, vol. 13-7, pp. 547$549,1988$.

[6] P Premaratne, C. C. Ko, "Retrieval Of Symmetrical Image Blur Using Zero Sheets", IEE Procee. Vision, Image and Signal Processing, vol. 148-1, pp. 65-69,2001.

[7] P. Premaratne and M. Premaratne, "Accelerated Iterative Blind Deconvolution of Still Images", Proc. of IEEE TENCON2003, 2003.

[7] P. Premaratne and C. C. Ko, "On the Human Visual Model for Blind Image Restoration", Proceedings of SCORED2001, pp. 52$57,2001$.

[8] P. Premaratne and C. C. Ko, "New Performance Metrics for Blind Image Restoration", Proceedings of IEEE 2001 In. Conf. IGARSS 2001, vol. 5, pp. 2322-2324, 2001. 